Elect. Comm. in Probab. 16 (2011), 165-173

ELECTRONIC

COMMUNICATIONS

in PROBABILITY

\title{
INDICATOR FRACTIONAL STABLE MOTIONS
}

\author{
PAUL JUNG ${ }^{1}$ \\ Department of Mathematics \\ Sogang University \\ Shinsu-dong, Mapo-gu \\ Seoul, South Korea 121-742 \\ email: pjung@sogang.ac.kr
}

Submitted October 16, 2010, accepted in final form Februrary 8, 2011

AMS 2000 Subject classification: 60G52, 60G22, 60G18

Keywords: fractional Brownian motion; random walk in random scenery; random reward schema; local time fractional stable motion; self-similar process; stable process

\begin{abstract}
Using the framework of random walks in random scenery, Cohen and Samorodnitsky (2006) introduced a family of symmetric $\alpha$-stable motions called local time fractional stable motions. When $\alpha=2$, these processes are precisely fractional Brownian motions with $1 / 2<H<1$. Motivated by random walks in alternating scenery, we find a complementary family of symmetric $\alpha$-stable motions which we call indicator fractional stable motions. These processes are complementary to local time fractional stable motions in that when $\alpha=2$, one gets fractional Brownian motions with $0<H<1 / 2$.
\end{abstract}

\section{Introduction}

There are a plethora of integral representations for Fractional Brownian motion (FBM) with Hurst parameter $H \in(0,1)$, and not surprisingly there are several generalizations of these integral representations to stable processes. These generalizations are often called fractional symmetric $\alpha$ stable ( $\alpha \alpha$ ) motions, with $0<\alpha<2$, and they can be considered analogs of FBM. Two common fractional $S \alpha S$ motions include linear fractional stable motion (L-FSM) and real harmonizable fractional stable motion (RH-FSM).

In [CS06], a new generalization of FBM, $H>1 / 2$, called local time fractional stable motion (LTFSM) was introduced. LT-FSM is particularly interesting because it is a subordinated process (this terminology is taken from Section 7.9 of [ST94] and should not be confused with subordination in the sense of time-changes). Subordinated processes are processes constructed from integral representations with random kernels, or said another way, where the stable random measure (of the integral representation) has a control measure related in some way to a probability measure of some other stochastic process (see Section 2 below). We note that subordinated processes are examples of what are known in the literature as doubly stochastic models.

${ }^{1}$ SUPPORTED IN PART BY SOGANG UNIVERSITY RESEARCH GRANT 200910039 
In this work we introduce another subordinated process which can be considered a natural extension of LT-FSM to $H<1 / 2$. The processes we consider have random kernels of a very simple type, namely the indicator function

$$
1_{\left[A_{0}, A_{t}\right]}(x) \quad\left(\left[A_{s}, A_{t}\right]:=\left[A_{t}, A_{s}\right] \text { if } A_{t}<A_{s}\right)
$$

with respect to some self-similar stationary increment (SSSI) process $A_{t}$. As such we call these processes indicator fractional stable motions (I-FSM).

I-FSM's relation to LT-FSM comes from the idea that the indicator function of a real-valued process $A_{t}$ can be thought of as an alternating version of the local time of $A_{t}$ in the following way. Suppose $S_{n}$, with $S_{0}=0$, is a discrete-time simple random walk on $\mathbb{Z}$. If $e$ is the edge between $k$ and $k+1$, then the discrete local time of $S_{n}$ at $e$ is the total number of times $S_{n}$ has gone from either $k$ to $k+1$ or from $k+1$ to $k$, up to time $n$. Now, instead of totaling the number of times $S_{n}$ crosses over edge $e$, one can consider the parity of the number of times $S_{n}$ crosses $e$ up to time $n$. The parity of the discrete local time at edge $e$ up to time $n$ is odd if and only if $e$ is between 0 and $S_{n}$. Thus, heuristically, the edges which contribute to an "alternating local time" are those edges which lie between 0 and $A_{t}$. This heuristic is discussed more rigorously in [JM11].

We can generalize the motivational discrete model to all random walks on $\mathbb{Z}$. In this case, when $S_{n}$ goes from $x$ to $y$ on a given step, it "crosses" all edges in between. In terms of the discrete local time, we heuristically think of the random walk as having spent a unit time at all edges between $x$ and $y$ during that time-step.

The first question one must ask is: are these new stable processes a legitimate new class of processes or are they just a different representation of L-FSMs and/or RH-FSMs? Using characterizations of the generating flows for the respective processes (see Section 3 below), [CS06] showed that the class of LT-FSMs is disjoint from the classes of RH-FSMs and L-FSMs. Following their lead, we use the same characterizations to show that when the (discretized) subordinating process $\left\{A_{n}\right\}_{n \in \mathbb{N}}$ is recurrent, the class of I-FSMs is also disjoint from the two classes, RH-FSMs and L-FSMs. Since I-FSMs and LT-FSMs have disjoint self-similarity exponents when $1<\alpha<2$, these two classes of processes are also disjoint when $1<\alpha<2$. For $\alpha<1$, the class of I-FSMs has a strictly larger self-similarity range than the class of LT-FSMs.

The outline of the rest of the paper is as follows. In the next section we define I-FSMs and show that they are S $\alpha$ S-SSSI processes. In Section 3 we give the necessary background concerning generating flows and characterizations with respect to them. In Section 4, we give the classification of I-FSMs according to their generating flows along with a result on the mixing properties of the stable noise associated with an I-FSM.

\section{Indicator fractional stable motions}

Let $m$ be a $\sigma$-finite measure on a measurable space $(B, \mathscr{B})$, and let

$$
\mathscr{B}_{0}=\{A \in \mathscr{B}: m(A)<\infty\} .
$$

Definition 2.1. A S $\alpha \mathrm{S}$ random measure $M$ with control measure $m$ is a $\sigma$-additive set function on $\mathscr{B}_{0}$ such that for all $A_{i} \in \mathscr{B}_{0}$

1. $M\left(A_{1}\right) \sim S_{\alpha}\left(m\left(A_{1}\right)^{1 / \alpha}\right)$

2. $M\left(A_{1}\right)$ and $M\left(A_{2}\right)$ are independent whenever $A_{1} \cap A_{2}=\emptyset$ 
where $S_{\alpha}(\sigma)$ is a $S \alpha S$ random variable with scale parameter $\sigma$ (see Section 3.3 of [ST94] for more details).

Another way to say the second property above is to say that $M$ is independently scattered.

For context, let us first define LT-FSM. Throughout this paper

$$
\lambda:=\text { Lebesgue measure on } \mathbb{R} .
$$

Let $\left(\Omega^{\prime}, \mathscr{F}^{\prime}, \mathbf{P}^{\prime}\right)$ support a subordinating process $A_{t} . A_{t}$ is either a FBM- $H^{\prime}$ or a S $\beta$ S-Levy motion, $\beta \in(1,2]$, with jointly continuous local time $L_{A}(t, x)\left(\omega^{\prime}\right)$. By self-similarity, $A_{0}=0$ almost surely. Suppose a $S \alpha S$ random measure $M$ with control measure $\mathbf{P}^{\prime} \times \lambda$ lives on some other probability space $(\Omega, \mathscr{F}, \mathbf{P})$. An LT-FSM is a process

$$
X_{A}^{H}(t):=\int_{\Omega^{\prime}} \int_{\mathbb{R}} L_{A}(t, x)\left(\omega^{\prime}\right) M\left(d \omega^{\prime}, d x\right), t \geq 0,
$$

where $X_{A}^{H}(t)$ is a S $\alpha$ S-SSSI process with self-similarity exponent $H=1-H^{\prime}+H^{\prime} / \alpha$ and $H^{\prime}$ is the self-similarity exponent of $A_{t}$ (see Theorem 3.1 in [CS06] and Theorem 1.3 in [DGP08]).

We now define I-FSM which is the main subject of this work. Let $\left(\Omega^{\prime}, \mathscr{F}^{\prime}, \mathbf{P}^{\prime}\right)$ support $A_{t}$, a nondegenerate S $\beta$ S-SSSI process with $\beta \in(1,2]$ and self-similarity exponent $H^{\prime} \in(0,1)$ (again by self-similarity $A_{0}=0$ almost surely). Suppose a $S \alpha \mathrm{S}$ random measure $M$ with control measure $\mathbf{P}^{\prime} \times \lambda$ lives on some other probability space $(\Omega, \mathscr{F}, \mathbf{P})$.

An indicator fractional stable motion is a process

$$
Y_{A}^{H}(t):=\int_{\Omega^{\prime}} \int_{\mathbb{R}} 1_{\left[0, A_{t}\left(\omega^{\prime}\right)\right]}(x) M\left(d \omega^{\prime}, d x\right), t \geq 0 .
$$

A nice observation is that the finite dimensional distributions of the process do not change if we replace the kernel $1_{\left[0, A_{t}\left(\omega^{\prime}\right)\right]}(x)$ with $\operatorname{sign}\left(A_{t}\left(\omega^{\prime}\right)\right) 1_{\left[0, A_{t}\left(\omega^{\prime}\right)\right]}(x)$ :

$$
\begin{aligned}
& \sum_{j=1}^{n} \theta_{j} \int_{\Omega^{\prime}} \int_{\mathbb{R}} \operatorname{sign}\left(A_{t_{j}}\left(\omega^{\prime}\right)\right) 1_{\left[0, A_{t_{j}}\left(\omega^{\prime}\right)\right]}(x) M\left(d \omega^{\prime}, d x\right) \\
= & \sum_{j=1}^{n} \theta_{j} \int_{\Omega^{\prime}} \int_{\mathbb{R}^{+}} 1_{\left\{\omega^{\prime}: A_{t_{j}}\left(\omega^{\prime}\right)>0\right\}} 1_{\left[0, A_{t_{j}}\left(\omega^{\prime}\right)\right]}(x) M\left(d \omega^{\prime}, d x\right) \\
& +\sum_{j=1}^{n} \theta_{j} \int_{\Omega^{\prime}} \int_{\mathbb{R}^{-}}-1_{\left\{\omega^{\prime}: A_{t_{j}}\left(\omega^{\prime}\right)<0\right\}} 1_{\left[0, A_{t_{j}}\left(\omega^{\prime}\right)\right]}(x) M\left(d \omega^{\prime}, d x\right) \\
\stackrel{d}{=} & \sum_{j=1}^{n} \theta_{j} \int_{\Omega^{\prime}} \int_{\mathbb{R}} 1_{\left[0, A_{t_{j}}\left(\omega^{\prime}\right)\right]}(x) M\left(d \omega^{\prime}, d x\right) .
\end{aligned}
$$

where the last line holds since $M$ is both symmetric and independently scattered.

The reason that this is helpful is because the equality

$$
\operatorname{sign}\left(A_{t}\right) 1_{\left[0, A_{t}\right]}(x)=\left(A_{t}-x\right)_{+}^{0}-(-x)_{+}^{0}
$$

makes it intuitively clear that the increments of $Y_{A}^{H}(t)$ are stationary. 
We note that both LT-FSM and I-FSM can technically be extended to the case where $A_{t}$ has selfsimilarity exponent $H^{\prime}=1$. In these degenerate cases, the kernels for LT-FSM and I-FSM coincide becoming the non-random family of functions $\left\{1_{[0, t]}\right\}_{t \geq 0}$ thereby giving us

$$
\int_{\mathbb{R}} 1_{[0, t]} M(d x), t \geq 0 .
$$

These are the $S \alpha$ L Levy motions with $\alpha \in(0,2)$.

Theorem 2.2. The process $Y_{A}^{H}(t)$ is a well-defined SaS-SSSI process with self-similarity exponent $H=H^{\prime} / \alpha$.

Proof. We start by noting that

$$
\begin{aligned}
\int_{\Omega^{\prime}} \int_{\mathbb{R}}\left|1_{\left[0, A_{t}\left(\omega^{\prime}\right)\right]}(x)\right|^{\alpha} d x \mathbf{P}^{\prime}\left(d \omega^{\prime}\right) & =\mathbf{E}^{\prime} \int_{\mathbb{R}} 1_{\left[0, A_{t}\left(\omega^{\prime}\right)\right]}(x) d x \\
& =\mathbf{E}^{\prime}\left|A_{t}\right|<\infty
\end{aligned}
$$

where the finite expectation follows since $A_{t}$ is a $S \beta S$ process with $\beta>1$. This shows that $Y_{A}^{H}(t)$ is a well-defined $S \alpha$ S process (see Section 3.2 of [ST94] for details).

Recall that the control measure for $M$ is $\mathbf{P}^{\prime} \times \lambda$. Using the alternative kernel given in (4), by Proposition 3.4.1 in [ST94] we have for $\theta_{j} \in \mathbb{R}$ and times $t_{j}, s_{j} \in \mathbb{R}^{+}$:

$$
\begin{aligned}
& \operatorname{E} \exp \left(i \sum_{j=1}^{k} \theta_{j}\left(Y_{A}^{H}\left(t_{j}\right)-Y_{A}^{H}\left(s_{j}\right)\right)\right) \\
= & \exp \left(-\int_{\mathbb{R}} \mathbf{E}^{\prime}\left|\sum_{j=1}^{k} \theta_{j} \cdot \operatorname{sign}\left(A_{t_{j}}-A_{s_{j}}\right) 1_{\left[A_{s_{j}}, A_{t_{j}}\right]}(x)\right|^{\alpha} d x\right) .
\end{aligned}
$$

Note that if we had not used the alternative kernel given in (4), then the right-side above would have been more complicated.

Using (7), we have

$$
\begin{aligned}
& \operatorname{E} \exp \left(i \sum_{j=1}^{k} \theta_{j}\left(Y_{A}^{H}\left(t_{j}+h\right)-Y_{A}^{H}(h)\right)\right) \\
= & \exp \left(-\int_{\mathbb{R}} \mathbf{E}^{\prime}\left|\sum_{j=1}^{k} \theta_{j} \cdot \operatorname{sign}\left(A_{t_{j}+h}-A_{h}\right) 1_{\left[A_{h}, A_{t_{j}+h}\right]}(x)\right|^{\alpha} d x\right) \\
= & \exp \left(-\int_{\mathbb{R}} \mathbf{E}^{\prime}\left|\sum_{j=1}^{k} \theta_{j} \cdot \operatorname{sign}\left(A_{t_{j}}\right) 1_{\left[0, A_{t_{j}}\right]}(x)\right|^{\alpha} d x\right) \\
= & \operatorname{E} \exp \left(i \sum_{j=1}^{k} \theta_{j} Y_{A}^{H}\left(t_{j}\right)\right)
\end{aligned}
$$

where the second equality follows since $A_{t}$ has stationary increments. The above shows that $Y_{A}^{H}(t)$ has stationary increments. 
Using (7) once more, the self-similarity of $\left\{A_{t}\right\}_{t \geq 0}$, and the change of variables $y=c^{-H^{\prime}} x$, we obtain

$$
\begin{aligned}
\operatorname{Eexp}\left(i \sum_{j=1}^{k} \theta_{j} Y_{A}^{H}\left(c t_{j}\right)\right) & =\exp \left(-\int_{\mathbb{R}} \mathbf{E}^{\prime}\left|\sum_{j=1}^{k} \theta_{j} \cdot \operatorname{sign}\left(A_{c t_{j}}\right) 1_{\left[0, A_{c t}\right]}\right|^{\alpha} d x\right) \\
& =\exp \left(-c^{H^{\prime}} \int_{\mathbb{R}} \mathbf{E}^{\prime}\left|\sum_{j=1}^{k} \theta_{j} \cdot \operatorname{sign}\left(A_{t_{j}}\right) 1_{\left[0, A_{t_{j}}\right]}\right|^{\alpha} d y\right) \\
& =\operatorname{Eexp}\left(i \sum_{j=1}^{k} \theta_{j} c^{H^{\prime} / \alpha} Y_{A}^{H}\left(t_{j}\right)\right)
\end{aligned}
$$

Remarks.

1. For each fixed $0<\alpha<2$, I-FSM is a class of S $\alpha$ S-SSSI processes with self-similarity exponents $H$ in the feasibility range $0<H<1 / \alpha$. In particular, when $1<\alpha<2$, this range of feasible $H$ complements that of LT-FSM which has the feasibility range $1 / \alpha<H<1$. When $0<\alpha<1$, the feasibility range $0<H<1 / \alpha$ of I-FSM is strictly bigger than that of LT-FSM: $1<H<1 / \alpha$.

2. It is not hard to see that I-FSMs are continuous in probability since the subordinating process $A_{t}$ is SSSI and continuous in probability. However, it follows from Theorem 10.3.1 in [ST94] that I-FSMs are not sample continuous. This is intuitive since I-FSMs should have continuity properties similar to those of $S \alpha$ Levy motions since the latter have the form

$$
\int_{\mathbb{R}^{+}} 1_{[0, t]}(x) M(d x), t \geq 0
$$

where $M$ is a $\mathrm{S} \alpha \mathrm{S}$ random measure with Lebesgue control measure.

3. By Theorem 11.1.1 in [ST94] an I-FSM has a measurable version if and only if the subordinating process $A_{t}$ has a measurable version.

\section{Background: Ergodic properties of flows}

Throughout this section we suppose that $0<\alpha<2$. The general integral representations of $\alpha$-stable processes, of the type

$$
X(t)=\int_{E} f_{t}(x) M(d x), t \in T
$$

$(T=\mathbb{Z}$ or $\mathbb{R}$ ) are well-known (see the introduction of [Sam05]). Here $M$ is a $S \alpha \mathrm{S}$ random measure on $E$ with a $\sigma$-finite control measure $m$, and $f_{t} \in L^{\alpha}(E, m)$ for each $t$. We call $\left\{f_{t}(x)\right\}_{t \in T}$ a spectral representation of $\{X(t)\}$. 
Definition 3.1. A measurable family of functions $\left\{\phi_{t}\right\}_{t \in T}$ mapping $E$ onto itself and such that

1. $\phi_{t+s}(x)=\phi_{t}\left(\phi_{s}(x)\right)$ for all $t, s \in T$ and $x \in E$,

2. $\phi_{0}(x)=x$ for all $x \in E$

3. $m \circ \phi_{t}^{-1} \sim m$ for all $t \in T$

is called a nonsingular flow. A measurable family $\left\{a_{t}\right\}_{t \in T}$ is called a cocycle for the flow $\left\{\phi_{t}\right\}_{t \in T}$ if for every $s, t \in T$ we have

$$
a_{t+s}(x)=a_{s}(x) a_{t}\left(\phi_{s}(x)\right) \text { m-a.e. }
$$

In [Ros95] it was shown that in the case of measurable stationary $S \alpha S$ processes one can choose the (spectral) representation in (11) to be of the form

$$
f_{t}(x)=a_{t}(x)\left(\frac{d m \circ \phi_{t}}{d m}(x)\right)^{1 / \alpha} f_{0} \circ \phi_{t}(x)
$$

where $f_{0} \in L^{\alpha}(E, m),\left\{\phi_{t}\right\}_{t \in T}$ is a nonsingular flow, and $\left\{a_{t}\right\}_{t \in T}$ is a cocycle, for $\left\{\phi_{t}\right\}_{t \in T}$, which takes values in $\{-1,1\}$. Also, note that one may always assume the following full support condition:

$$
\operatorname{supp}\left\{f_{t}: t \in T\right\}=E .
$$

Henceforth we shall assume that $T=\mathbb{Z}$ and will write $f_{n}, \phi_{n}$, and $X(n)$. Note that in the discrete case we may always assume measurability of the process (see Section 1.6 of [Aar97]). Given a representation of the form (13), we say that $X(n)$ is generated by $\phi_{n}$.

In [Ros95] and [Sam05], the ergodic-theoretic properties of a generating flow $\phi_{n}$ are related to the probabilistic properties of the $\mathrm{S} \alpha \mathrm{S}$ process $X(n)$. In particular, certain ergodic-theoretic properties of the flow are found to be invariant from representation to representation.

In Theorem 4.1 of [Ros95] it was shown that the Hopf decomposition of a flow is a representationinvariant property of stationary $\mathrm{S} \alpha \mathrm{S}$ processes. Specifically, one has the disjoint union $E=C \cup D$ where the dissipative portion $D$ is the union of all wandering sets and the conservative portion $C$ contains no wandering subset. A wandering set is one such that $\left\{\phi_{n}(B)\right\}_{n \in \mathbb{Z}}$ are disjoint modulo sets of measure zero. Since $C$ and $D$ are $\left\{\phi_{n}\right\}$-invariant, one can decompose a flow by looking at its restrictions to $C$ and $D$, and the decomposition is unique modulo sets of measure zero. A nonsingular flow $\left\{\phi_{n}\right\}$ is said to conservative if $m(D)=0$ and dissipative if $m(C)=0$.

The following result appeared as Corollary 4.2 in [Ros95] and has been adapted to the current context:

Theorem 3.2 (Rosinski). Suppose $0<\alpha<2$. A stationary S $\alpha$ S process is generated by a conservative (dissipative, respectively) flow if and only if for some (all) measurable spectral representation $\left\{f_{n}\right\}_{n \in \mathbb{R}^{+}} \subset L^{\alpha}(E, m)$ satisfying (14), the sum

$$
\sum_{n \in \mathbb{Z}}\left|f_{n}(x)\right|^{\alpha}
$$

is infinite (finite) m-a.e. on $E$.

In [Sam05], another representation-invariant property of flows, the positive-null decomposition of stationary $S \alpha$ S processes, was introduced.

A subset $B \subset E$ is called weakly wandering if there is a subsequence with $n_{0}=0$ such that the sets $\left\{\phi_{n_{k}} B\right\}_{k \in \mathbb{N}}$ are disjoint modulo sets of measure zero. The null part $N$ of $E$ is the union of all weakly 
wandering sets, and the positive part $P$ contains no weakly wandering set. Note that the positive part of $E$ is a subset of the conservative part, i.e. $P \subset C$. Again, one can decompose $\left\{\phi_{n}\right\}$ by restricting to $P$ and $N$. This decomposition is unique modulo sets of measure zero, and Theorem 2.1 of [Sam05] states that the decomposition is representation-invariant modulo sets of measure zero. A null flow is one with $m(P)=0$ and a positive flow has $m(N)=0$. Note that dissipative flows are automatically null flows, however in the case of conservative flows, both positive and null flows are possible.

\section{Ergodic properties of indicator fractional stable noise}

Properties of a S $\alpha$ S-SSSI process $Y(t)$ are often deduced from its increment process $Z(n)=Y(n)-$ $Y(n-1), n \in \mathbb{N}$ called a stable noise. In this section, we study the ergodic-theoretic properties (which were introduced in the previous section) of indicator fractional stable noise (I-FSN) which we define as

$$
Z_{A}(n):=\int_{\Omega^{\prime}} \int_{\mathbb{R}} 1_{\left[0, A_{n}\left(\omega^{\prime}\right)\right]}(x)-1_{\left[0, A_{n-1}\left(\omega^{\prime}\right)\right]}(x) M\left(d \omega^{\prime}, d x\right), n \in \mathbb{N} .
$$

We note that in light of the proof of Theorem 2.2, one may deem it natural to instead use the kernel

$$
\operatorname{sign}\left(A_{n}\left(\omega^{\prime}\right)\right) 1_{\left[0, A_{n}\left(\omega^{\prime}\right)\right]}(x)-\operatorname{sign}\left(A_{n-1}\left(\omega^{\prime}\right)\right) 1_{\left[0, A_{n-1}\left(\omega^{\prime}\right)\right]}(x) .
$$

However, as seen in (4), the $\operatorname{sign}\left(A_{t}\right)$ has no affect on the distribution of the process and therefore has no affect on the distribution of its increments.

It is known that stationary $\mathrm{S} \alpha \mathrm{S}$ processes generated by dissipative flows are mixing [SRMC93]. Concerning conservative flows, Theorem 3.1 of [Sam05] states that a stationary $S \alpha S$ process is ergodic if and only if it is generated by a null flow, and examples are known of both mixing and non-mixing stationary $S \alpha S$ processes generated by conservative null flows (see Section 4 of [GR93]). Our next goal is to show that I-FSN is mixing which implies that its flow is either dissipative or conservative null. We first need a result which appeared as Theorem 2.7 of [Gro94]:

Lemma 4.1 (A. Gross). Suppose $X_{n}$ is some stationary $S \alpha S$ process, and assume $\left\{f_{n}\right\} \subset L^{\alpha}(E, m)$ is a spectral representation of $X_{n}$ with respect to the control measure $m$. Then $X_{n}$ is mixing if and only if for every compact $K \subset \mathbb{R}-\{0\}$ and every $\epsilon>0$,

$$
\lim _{n \rightarrow \infty} m\left\{x: f_{0} \in K,\left|f_{n}\right|>\epsilon\right\}=0 .
$$

Theorem 4.2. Indicator fractional stable noise is a mixing process.

Proof. Using the above lemma, it suffices to show that

$$
\lim _{n \rightarrow \infty}\left(\mathbf{P}^{\prime} \times \lambda\right)\left\{\left(\omega^{\prime}, x\right): x \in\left[0, A_{1}\right], x \in\left[A_{n}, A_{n+1}\right]\right\}=0,
$$

recalling that $\left[A_{n}, A_{n+1}\right]:=\left[A_{n+1}, A_{n}\right]$ whenever $A_{n+1}<A_{n}$.

Let $c_{i}$ be constants such that for all $M>0, \mathbf{P}^{\prime}\left(A_{1}>M\right)<c_{1} M^{-\beta}$ and

$$
\int_{M}^{\infty} \mathbf{P}^{\prime}\left(A_{1}>x\right) d x<c_{2} M^{-\beta+1}
$$


where $\beta>1$. Also, recall that $0<H^{\prime}<1$ is the self-similarity exponent of $A_{t}$. We have that

$$
\begin{aligned}
& \left(\mathbf{P}^{\prime} \times \lambda\right)\left\{\left(\omega^{\prime}, x\right): x \in\left[0, A_{1}\right], x \in\left[A_{n}, A_{n+1}\right]\right\} \\
= & \left(\mathbf{P}^{\prime} \times \lambda\right)\left\{\left(\omega^{\prime}, x\right):|x|>M, x \in\left[0, A_{1}\right], x \in\left[A_{n}, A_{n+1}\right]\right\} \\
& +\left(\mathbf{P}^{\prime} \times \lambda\right)\left\{\left(\omega^{\prime}, x\right):|x| \leq M, x \in\left[0, A_{1}\right], x \in\left[A_{n}, A_{n+1}\right]\right\} \\
\leq & 2 \int_{M}^{\infty} \mathbf{P}^{\prime}\left(A_{1}>x\right) d x+\left(\mathbf{P}^{\prime} \times \lambda\right)\left\{\left(\omega^{\prime}, x\right):|x| \leq M, x \in\left[A_{n}, A_{n+1}\right]\right\} \\
\leq & 2 c_{2} M^{-\beta+1}+2 M \sup _{x \in[-M, M]} \mathbf{P}^{\prime}\left\{\omega^{\prime}: x \in\left[A_{n}\left(\omega^{\prime}\right), A_{n+1}\left(\omega^{\prime}\right)\right]\right\} \\
\leq & 2 c_{2} M^{-\beta+1}+2 M \mathbf{P}^{\prime}\left(\left\{\left|A_{n}\right| \leq M\right\} \cup\left\{\left|A_{n+1}\right| \leq M\right\}\right) \\
& +2 M \mathbf{P}^{\prime}\left(\left\{A_{n}<-M, A_{n+1}>M\right\} \cup\left\{A_{n}>M, A_{n+1}<-M\right\}\right) \\
\leq & 2 c_{2} M^{-\beta+1}+4 M \mathbf{P}^{\prime}\left(\left|A_{1}\right| \leq M / n^{H^{\prime}}\right)+2 M \cdot 2 c_{1} M^{-\beta} .
\end{aligned}
$$

where the first inequality uses the symmetry of $A_{1}$. The second inequality uses (19), and the third inequality uses the fact that for $x \in[-M, M]$, the event $\left\{\omega^{\prime}: x \in\left[A_{n}\left(\omega^{\prime}\right), A_{n+1}\left(\omega^{\prime}\right)\right]\right\}$ is contained by the event that either $A_{n}$ or $A_{n+1}$ is in $[-M, M]$ or that $\left[A_{n}, A_{n+1}\right]$ (which we defined as equivalent to $\left[A_{n+1}, A_{n}\right]$ ) contains $[-M, M]$. The final inequality uses both self-similarity and stationarity of increments.

Since the right side of (20) can be made arbitrarily small by choosing $M$ and then $n$ appropriately, the result is proved.

Since I-FSN is mixing, it is generated by a flow which is either dissipative or conservative null. Our next result classifies the flow of I-FSN as conservative if almost surely

$$
\limsup _{n \rightarrow \infty} A_{n}=+\infty \quad \text { and } \quad \liminf _{n \rightarrow \infty} A_{n}=-\infty \quad \text { where } n \in \mathbb{N} .
$$

This holds, for example, when $A_{t}$ is a FBM or a S $\beta$ S Levy motion with $\beta>1$.

Theorem 4.3. If the subordinating process $A_{t}$ satisfies (21), then the indicator fractional stable noise, $\left\{Z_{A}(n)\right\}_{n \in \mathbb{Z}}$, is generated by a conservative null flow.

Proof. By (21), we have that $\mathbf{P}^{\prime}$-almost surely

$$
\begin{aligned}
& \sum_{n=0}^{\infty}\left|1_{\left[0, A_{n}\left(\omega^{\prime}\right)\right]}(x)-1_{\left[0, A_{n-1}\left(\omega^{\prime}\right)\right]}(x)\right|^{\alpha} \\
& =\sum_{n=0}^{\infty} 1_{\left[A_{n}\left(\omega^{\prime}\right), A_{n+1}\left(\omega^{\prime}\right)\right]}(x)=\infty \text { for every } x .
\end{aligned}
$$

Hence by Theorem 3.2 we have that $Z_{A}(n)$ is generated by a conservative flow. By Theorem 4.2 the flow is also null.

Remarks. 
1. When $A_{n}$ satisfies (21), the fact that I-FSMs are generated by conservative null flows implies they form a class of processes which are disjoint from the class of RH-FSMs (positive flows) and disjoint from the class of L-FSMs (dissipative flows). We have already seen that the classes of I-FSMs and LT-FSMs are disjoint when $1<\alpha<2$ due to their self-similarity exponents.

2. Another useful property of conservative flows comes from Theorem 4.1 of [Sam04]: If $Z_{A}(n)$ is generated by a conservative flow, then it satisfies the following extreme value property:

$$
n^{-1 / \alpha} \max _{j=1, \ldots n} Z_{A}(n) \stackrel{p}{\rightarrow} 0 .
$$

\section{Acknowledgements}

We wish to thank an anonymous referee for a careful reading and helpful comments.

\section{References}

[Aar97] J. Aaronson. An introduction to infinite ergodic theory. AMS, 1997. MR1450400

[CS06] S. Cohen and G. Samorodnitsky. Random rewards, fractional Brownian local times and stable self-similar processes. The Annals of Applied Probability, 16(3):1432-1461, 2006. MR2260069

[DGP08] C. Dombry and N. Guillotin-Plantard. Discrete approximation of a stable self-similar stationary increments process. Bernoulli, 2008. MR2546804

[GR93] A. Gross and J.B. Robertson. Ergodic properties of random measures on stationary sequences of sets. Stochastic Processes and their Applications, 46(2):249-265, 1993. MR1226411

[Gro94] A. Gross. Some mixing conditions for stationary symmetric stable stochastic processes. Stochastic Processes and their Applications, 51(2):277-295, 1994. MR1288293

[JM11] P. Jung and G. Markowsky. Scaling limits of random walks in alternating scenery and a construction of fractional Brownian motion. Preprint, 2011.

[Ros95] J. Rosinski. On the structure of stationary stable processes. The Annals of Probability, 23(3):1163-1187, 1995. MR1349166

[Sam04] G. Samorodnitsky. Extreme value theory, ergodic theory and the boundary between short memory and long memory for stationary stable processes. Annals of Probability, 32(2):1438-1468, 2004. MR2060304

[Sam05] G. Samorodnitsky. Null flows, positive flows and the structure of stationary symmetric stable processes. Annals of probability, 33(5):1782-1803, 2005. MR2165579

[SRMC93] D. Surgailis, J. Rosinski, V. Mandrekar, and S. Cambanis. Stable mixed moving averages. Probability Theory and Related Fields, 97(4):543-558, 1993. MR1246979

[ST94] G. Samorodnitsky and M.S. Taqqu. Stable non-Gaussian random processes: stochastic models with infinite variance. Chapman \& Hall/CRC, 1994. MR1280932 